\title{
Modeling the impact of sub-grid scale emission variability on upper-air concentration
}

\author{
S. Galmarini ${ }^{1}$, J.-F. Vinuesa ${ }^{1}$, and A. Martilli $^{2}$ \\ ${ }^{1}$ European Commission - DG Joint Research Centre, Institute for Environment and Sustainability, 21020 Ispra, Italy \\ ${ }^{2}$ CIEMAT, Madrid, Spain
}

Received: 31 July 2007 - Published in Atmos. Chem. Phys. Discuss.: 20 August 2007

Revised: 21 November 2007 - Accepted: 7 December 2007 - Published: 15 January 2008

\begin{abstract}
The long standing issue of sub-grid emission heterogeneity and its influence to upper air concentration is addressed here and a subgrid model proposed. The founding concept of the approach is the assumption that average emission act as source terms of average concentration, emission fluctuations are source for the concentration variance. The model is based on the derivation of the sub-grid contribution of emission and the use of the concentration variance equation to transport it in the atmospheric boundary layer. The model has been implemented in an existing mesoscale model and the results compared with Large-Eddy Simulation data for ad-hoc simulation devised to test specifically the parametrization. The results show an excellent agreement of the models. For the first time a time evolving error bar reproducing the sub-grid scale heterogeneity of the emissions and the way in which it affects the concentration has been shown. The concentration variance is presented as an extra attribute to better define the mean concentrations in a Reynolds-average model. The model has applications from meso to global scale and that go beyond air quality.
\end{abstract}

\section{Introduction}

There is an interesting exercise that we invite every air quality modeler to perform sure that the majority of them do it regularly or have done it at least once. Take a detailed map of highly inhabited and industrialized area, as there are many around the world, and draw over it a scaled grid cell of the size normally used in air quality simulations. In spite of the fact that these days a grid resolution for a mesoscale air quality simulation can confidently get to the order of few kilometers, what surprises is to see in detail the sheer variety of sources that can fall within the depicted unit surface. Variety

Correspondence to: S. Galmarini

(stefano.galmarini@jrc.it) in terms of shapes (point, area, line), quality of the emission (type of primary pollutants emitted), quantity or intensity of the emission (individual households versus vehicle fleet versus industry), time evolution of the emission (vehicle fleet versus industrial activities), spatial inhomogeneity.

Air quality models normally have as available input average emission rates for various primary pollutants which account for the volume averaged quantity of mass released per unit time. No other information takes into account the fact that for example a large amount of mass can be emitted by a small portion of the grid surface or by several sources scattered around it. We will refer to this as sub-grid emission heterogeneity. The emission heterogeneity can be seen quite easily by disaggregating an emission inventory for a specific species over a mesh smaller than the one used for atmospheric transport. Within each element of the mesh different surfaces will emit different amounts of mass. The emission heterogeneity is completely lost in the volume averaging process performed within numerical models and no indication on the sub-grid emission variability is therefore transferred to the upper atmospheric layers. So far no indication has been given on the relevance of this information to upper atmospheric levels and its impact on upper air concentration.

This is the problem that we will try to solve in this study. We will propose a method to account for the sub-grid emission heterogeneity and the way to transfer the information to the upper atmosphere. We will not address here the role of the spatial distribution of the different sources in the sense of the actual position they occupy within the transport model cell but rather the fact that within the grid we consider the existence of a fine emission structure. The research questions that we wish to address in this paper are the following:

- Is it possible to take into account the effect of the subgrid scale source heterogeneity in a meso- or larger scale model?

- What would be the minimum set of information

Published by Copernicus Publications on behalf of the European Geosciences Union. 
necessary to account for the contribution of the individual sub-grid emitting surfaces?

- What if associated to the mean emission rate one would have the first moment statistics, namely the emission variance?

- In what ways this additional, though not detailed information, can be used to improve the effect of emission on air concentration?

- Provided that a method is found to transfer the information on the emission sub-grid variability to the upper atmospheric layers, what is the distance in the vertical at which it can not be distinguished any more and therefore can be disregarded?

- Is the information on emission heterogeneity going to be transported at long distances downwind? Therefore is horizontal advection a relevant process in the information transfer, to what scale is it so?

To our knowledge no previous investigation has tackled these issues.

\section{Parameterizing sub-grid scale emission variability}

Let us formulate the problem: given an undefined non reactive pollutant, which is emitted by a series of surfaces within a model grid with different rates, is there a way to transfer the information on the sub-grid emission heterogeneity to the atmospheric concentration of the species?

It is appropriate at this stage to specify that when we generically refer to a model we refer to Reynolds-Average NavierStokes (RANS) models or equivalently Reynolds-Averaged (RA) models. Within this classification fall all the atmospheric models ranging from meso- to global scale. They may solve explicitly the dynamics on top of the tracer transport (RANS) or simply using off-line meteorology to model atmospheric transport (RA). The feature common to these models is the fact that the variables are grid and time averaged and no information is available or deducible about their sub-grid scale behavior unless parameterized. All the considerations that will follow therefore apply to any model falling in these two classes and which are normally used for air quality analysis from the meso- to the global scale.

The founding concept of our approach toward the parametrization of sub-grid scale emission heterogeneity is based on the fact that turbulent motion in the atmospheric boundary layer is responsible for the creation and dissipation of variability around the concentration mean. Turbulence creates and transports it at higher levels as well as horizontally (through the mean wind) even in the case of a uniform surface emission. We will use this concept to try to link formally the emission variability at surface with the concentration variability in the boundary layer.
In the atmospheric boundary layer (ABL) the action of turbulence in dispersing a generic pollutant released at the surface or entrained at its top, is represented by the concept of concentration variance. Since the fluctuations of the species concentration due to the turbulent motion cannot be resolved explicitly, it is normal practice to represent them in terms of statistical variance $\left(\overline{c^{\prime 2}}\right)$, i.e., the square of the standard deviation around the mean concentration. The time evolution of the variance in the ABL reads (e.g. Stull, 1988; Garratt, 1994):

$\frac{\partial \overline{c^{\prime 2}}}{\partial t}+\overline{u_{j}} \frac{\partial \overline{c^{\prime 2}}}{\partial x_{j}}=-2 \overline{u_{j}^{\prime} c^{\prime}} \frac{\partial \bar{c}}{\partial x_{j}}-\frac{\partial \overline{u_{j}^{\prime} c^{\prime 2}}}{\partial x_{j}}-2 \epsilon_{c}$,

where from left to right the equation terms account for:

1 Time evolution of the concentration variance,

2 Advection or transport term,

3 Production term due to turbulent motions within the mean concentration gradient,

4 Turbulent transport of variance,

5 Dissipation.

For the sake of simplicity we will consider here the one dimensional version of Eq. (1) in conditions of horizontal homogeneity, namely:

$\frac{\partial \overline{c^{\prime 2}}}{\partial t}=-2 \overline{w^{\prime} c^{\prime}} \frac{\partial \bar{c}}{\partial z}-\frac{\partial \overline{w^{\prime} c^{\prime 2}}}{\partial z}-2 \epsilon_{c}$,

all the consideration done hereafter can be extended to the remaining two spatial dimensions. There are several conventional and well tested ways in which the unclosed terms of Eq. (2) (terms 3,4,5 of Eq. 1) can be parameterized. To avoid distracting the attention of our readers from the actual central topic of this paper the parameterizations adopted are presented in the Appendix.

The concentration variance equation will be used as carrier of the information on the emission heterogeneity from the surface to the upper atmospheric layers. We need therefore to create a connection between the concentration variance equation and the sub-grid emission. To do that we first express emissions in sub-grid terms. In general let us assume $E_{i}$ as the individual emission from a sub-grid scale surface within the grid-cell of a RANS model. $E_{i}$ is defined as:

$E_{i}=\frac{M_{i}}{a_{i} T}$,

where $M_{i}$ is the amount of mass emitted per unit time $T$ and $a_{i}$ is a sub-grid surface that satisfies the condition:

$a_{i}<A$, 
where $A$ is the surface of the grid cell of the RANS model. The other conditions on the sub-grid emitting surfaces is that given $N$ the total number of sub-grid emitting surfaces:

$A=\sum_{i=1}^{N} a_{i}$

At this point we will assume that we can decompose the emission into:

$E_{i}=\widehat{E}+E_{i}^{\prime \prime}$

where:

$\widehat{E}=\frac{1}{A} \sum_{i=1}^{N} a_{i} E_{i}$

In the case in which the sub-grid scale emitting surfaces $a_{i}$ are of equal size $(a)$ for all $i$ 's (which is normally what is obtained as a result of the disaggregation of an emission inventory at a scale smaller than the RANS model grid size) the average can also be calculated as:

$\widehat{E}=\frac{1}{N a} \sum_{i=1}^{N} \frac{M_{i}}{T}$

The different symbols used in Eq. (3) to identify average and fluctuation compared to the notation of Eqs. (1) and (2) are due to the fact that the average and consequently the fluctuation should be considered over a surface whereas the overbar and the single accent are defined relative to a volume. We could at this stage imagine that the emissions (average and fluctuation) pertain to a volume of air sitting right above the surface. Eventually one could assume that the vertical extension of such volume could go as far as the first numerical grid cell. As a matter of fact this is implicitly done whenever a tracer is injected into a numerical model. Through this assumption we can now directly relate the two averaging operators $(\bar{E}$ and $\widehat{E})$ so that the linearization of the $E$ now reads:

$E_{i}=\bar{E}+E_{i}^{\prime}$,

with $E_{i}, \bar{E}$, and $E_{i}^{\prime}$ now expressed as [mass $L^{-3} T^{-1}$.

At this stage we will separate the contribution of the emission in the sense that the average emission $(\bar{E})$ will contribute to the average concentration $(\bar{c})$ according to the classical approach, while the emission fluctuation $\left(E_{i}^{\prime}\right)$ contributes to the concentration fluctuation $\left(c^{\prime}\right)$. Therefore we re-derive the concentration variance equation taking into account the emission fluctuation as an extra production term. Following the classic derivation we first derive the conservation equation of the concentration fluctuation:

$$
\frac{\partial c^{\prime}}{\partial t}=w^{\prime} \frac{\partial \bar{c}}{\partial z}+\frac{\partial w^{\prime} c^{\prime}}{\partial z}+E^{\prime}+v_{c} \frac{\partial^{2} c^{\prime}}{\partial^{2} z},
$$

to which we have added the contribution of the emissions fluctuations. In the latter $v_{c}$ stands for molecular diffusion.
Then we multiply both sides with $2 c^{\prime}$ and apply derivation rules to obtain after averaging:

$\frac{\partial \overline{c^{\prime 2}}}{\partial t}=-2 \overline{w^{\prime} c^{\prime}} \frac{\partial \bar{c}}{\partial z}-\frac{\partial \overline{w^{\prime} c^{\prime 2}}}{\partial z}+2 \overline{c^{\prime} E^{\prime}}-2 \epsilon_{c}$.

The presence of the emission fluctuation has generated the extra term $2 \overline{c^{\prime} E^{\prime}}$ that we will define concentration-emission covariance (CEC) term. The interesting aspect of Eq. (8) resides in the fact that we have added an extra term that represents a source of variance and that is directly connected to the emission fluctuations. All the other terms in the equation remain unchanged as one can see by comparing Eq. (8) with (2) and therefore the parameterizations presented in Appendix are still valid. The new variance equation accounts for a source term while the other terms create it, transport it, disperse it and dissipate it as expected from the turbulent motion thus acting as carriers of the information to the upper layers.

The problem now is how to close the CEC term in order to make the equation solvable. If we start from the consideration that the correlation coefficient between the concentration and the emission is formally given by:

$r=\frac{\overline{c^{\prime} E^{\prime}}}{\left(\overline{E^{\prime 2}}\right)^{1 / 2}\left(\overline{c^{\prime 2}}\right)^{1 / 2}}$,

we can derive a simple and straight forward parametrization of the CEC term as:

$\overline{c^{\prime} E^{\prime}}=r\left(\overline{E^{\prime 2}}\right)^{1 / 2}\left(\overline{c^{\prime 2}}\right)^{1 / 2}$

With expression (10) the closure has been transferred to the correlation coefficient $r$ which will be defined in the proceedings. The closure adopted has formally restricted the range of variability of the closure constant $r$ between 0 and 1 by definition as negative values would be counter intuitive. In other words provided sufficient level of turbulence intensity, close to the surface the concentration variance can only be expected to increase with the increase of the emission fluctuation. In any case this will be a hypothesis that needs to be verified and a more precise functional relationship for $r$ needs to be provided. Once $r$ will be defined Eq. (7) will be closed. In fact the other term in Eq. (10), $\overline{E^{\prime 2}}$, can be calculated very simply from the emission inventory as boundary condition of our problem. It will be the main character of this play and a key parameter toward a better estimate of boundary layer concentration levels. Following the derivation of the average emission given by Eq. (4) the formula to calculate the volume averaged emission variance in the case of generic sub-grid emitting surfaces $a_{i}$ reads:

$\overline{E^{\prime 2}}=\left[\frac{1}{A} \sum_{i=1}^{N} a_{i}\left(\widehat{E}-E_{i}\right)^{2}\right] \Delta z$, 
where $\Delta z$ is the extension of the first grid point in the vertical. While for surfaces with equal size it is:

$\overline{E^{\prime 2}}=\left[\frac{1}{N} \sum_{i=1}^{N}\left(\widehat{E}-\frac{M_{i}}{T}\right)^{2}\right] \Delta z$

It is worth to notice that the $\left(\overline{E^{\prime 2}}\right)^{1 / 2}$ corresponds to the standard deviation of the emission and represents the simplest way in which the sub-grid emission heterogeneity can be represented. It should be clear that the smaller the surfaces in which the emission contributions are broken up below the RANS model grid size the higher the variance and therefore the higher the detail in which the contribution will be accounted for. We have implicitly assumed that the emissions have a gaussian distribution around the mean value which may sound as an over simplification but effectively it is a great improvement with respect to the past.

To summarize the parameterization consists of calculating the sub-grid emission variability from the emission inventory in terms of emission variance and to solve the concentration variance equation in which the extra contribution to the concentration fluctuation has been included and closed as from Eq. (10). In this way going back to a description provided above, the mean emission acts as source term of the mean concentration and through Eq. (10) the emission variance effectively modulated by the correlation coefficient acts as source term to the concentration variance equation. Average concentration emission and concentration standard deviation will be used simultaneously to describe the evolution of the tracer in the atmosphere.

\section{Reynolds-averaged modeling vs. Large eddy simula- tion}

The way in which we are going to test the parametrization presented in the previous section is by means of Large-Eddy Simulations (LES) of controlled emission cases and a three dimensional Reynolds Averaged Navier-Stokes (RANS) model. Large-eddy simulation has been selected as it guarantees a detailed representation of the turbulent flow and dispersion in the atmospheric boundary layer ranging from the peak of maximum spectral energy down to the dissipation scale. In such controlled flow conditions we are able to define specific, detailed and controlled emission scenarios. Any real case application selected to verify the parameterization will allow only an indirect verification through mean variables as the concentration variances are never measured over long periods of time and more importantly never over large areas. LES, on the contrary, allows us a thorough assessment of the closure.

All the large-eddy simulations are run on a domain size corresponding to few grid cells of a mesoscale model. Namely the atmospheric flow contained in a volume of $12 \mathrm{~km} \times 12 \mathrm{~km} \times 1500 \mathrm{~m}$ is simulated by LES by means of
$120 \times 120 \times 60$ grid points whereas the RANS model will use $4 \times 4 \times 60$ cells in total accounting for $3 \times 3 \mathrm{~km}$ in horizontal resolution. The coarse resolution of the RANS model has been selected on purpose to push to the limit the parametrization. Figure 1 shows a schematic representation of the two domains. Slab averaging operations are performed at all vertical levels to make the LES results comparable to the ones of a RANS. The slabs over which the averages are taken correspond to the dimensions of the individual RANS model cells. In Fig. 1, 6 of the 16 cells of the RANS model (corresponding to 16 LES sub-domains) have been labeled A through F in order to facilitate the analysis of the results. The 6 cells are representative in terms of source location and flow direction.

\subsection{The models and their set-ups}

The LES model used is the one developed by Cuijpers and Duynkerke (1993), Siebesma and Cuijpers (1995), Cuijpers and Holtslag (1998) and Vilà-Guerau de Arellano and Cuijpers (2000). The model has evolved over the years and has been successfully used to study many different processes in the ABL, from cloud dynamics (e.g., Cuijpers and Duynkerke, 1993; Siebesma and Cuijpers, 1995; Siebesma and Holtslag, 1996; van Zanten et al., 1999; Siebesma et al., 2003; Neggers et al., 2003), chemical reaction and radioactive decay in atmospheric turbulent environments (e.g., Meeder and Nieuwstadt, 2000; Vinuesa and Vilà-Guerau de Arellano, 2003; Jonker et al., 2004; Vinuesa and Vilà-Guerau de Arellano, 2005; Vinuesa and Galmarini, 2007), the influence of clouds on atmospheric chemistry (e.g., Vilà-Guerau de Arellano and Cuijpers, 2000; Jonker et al., 2004; VilàGuerau de Arellano et al., 2005), plume dispersion (e.g. Nieuwstadt, 1992a,b; Meeder and Nieuwstadt, 2000; Dosio et al., 2003; Vilà-Guerau de Arellano et al., 2004; Dosio et al., 2005; Dosio and Vilà-Guerau de Arellano, 2006), stable BL (e.g., Galmarini et al., 1998; Beare et al., 2006). For a detailed description of the model we refer the reader the above mentioned references.

A full RANS model has been selected for the sake of completeness of this study, we could more simply have opted for a pure transport model and use the LES flow to run the dispersion. The three-dimensional RANS model is the mesoscale model described in detail by Martilli (2002). The concentration mean equation for the transport of a passive scalar together with the full three-dimensional formulation of the variance Eq. (8) have been added to the original model formulation for the sake of this study.

The LES simulation runs for $3 \mathrm{~h}$ with maximum timestep used in the calculations is $0.5 \mathrm{~s}^{-1}$. The surface sensible heat flux is set at $0.05 \mathrm{Kms}^{-1}$. A constant westerly wind of $5 \mathrm{~ms}^{-1}$ has been imposed. The initial potential temperature profile has a constant value of $288 \mathrm{~K}$ below $662.5 \mathrm{~m}$ and increases by $6 \mathrm{~K}$ above $712.5 \mathrm{~m}$. The surface roughness length $z_{0}$ is set to $0.01 \mathrm{~m}$. Periodic lateral boundary conditions are assumed. At the end of the first hour temperature and wind 

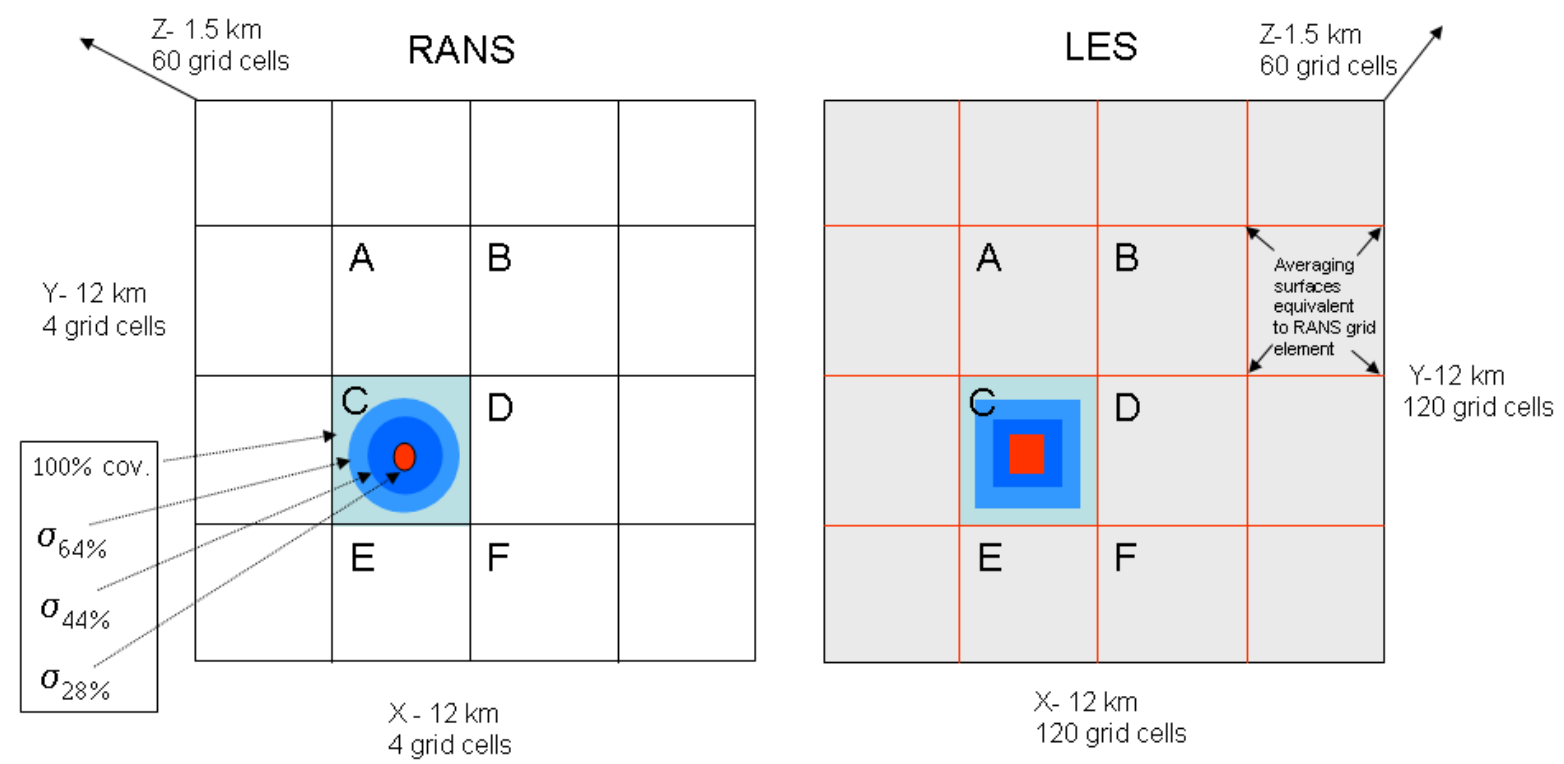

Fig. 1. Schematic representation of the the two model computational grids and emission scenarios. Left panel: RANS grid. Letters A to F identify grid where results will be shown. Cell C contains the various emission patterns ranging from $100 \%$ coverage of the grid to $28 \%$ coverage. Righ panel LES grid. In red the sub-domains corresponding to the RANS grid over which the LES results are averaged.

profiles are provide to the RANS models as initial condition for its simulation. LES data are averaged over the last simulation hour before they are compared with the RANS results.

\subsection{Flow and turbulence simulations}

Before we analyze the tracer release set up and the results relating to the scalar variance we present here the results of the flow simulation comparison of RANS vs LES. Figure 2a$\mathrm{d}$ show the vertical profile of $\mathrm{M}$ (total wind) and potential temperature $\Theta$, turbulent kinetic energy and heat flux respectively calculated by the LES and RANS for sub-domain. The plots relate to sub-grid cell C only (see Fig. 1) as the same result is obtained in the others. The RANS model is able to simulate with a relatively high degree of accuracy the wind speed and direction simulated by the LES. Small discrepancies are found for $\mathrm{M}$ that shows a slight systematic underestimation of the LES profile. On the temperature the agreement with the LES is very good in spite of a slightly higher diffusivity of the RANS model at the boundary layer top that lead to a different slope in the temperature profile and the inversion. The turbulence intensity and distribution is presented in Fig. $2 \mathrm{c}$ by the profile of the turbulent kinetic energy. Even for this variable the comparison between the two models can be considered satisfactory in spite of differences of curvature in the vertical profile and an underestimation at the boundary layer top. The heat flux is modeled very well by the RANS model including the negative flux component at the boundary layer top.

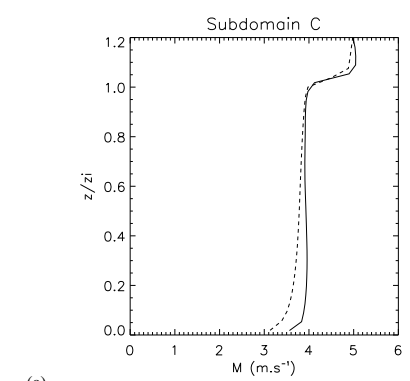

(a)

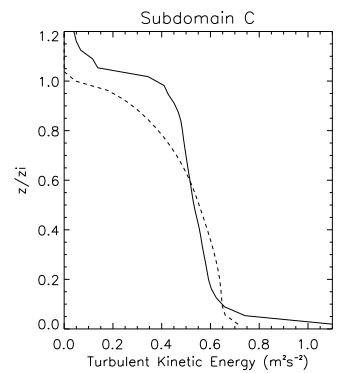

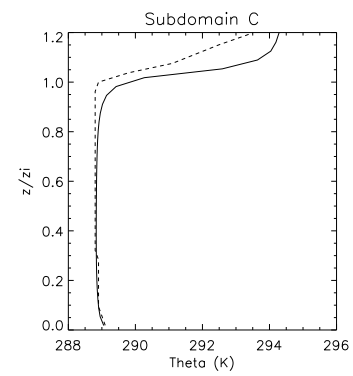

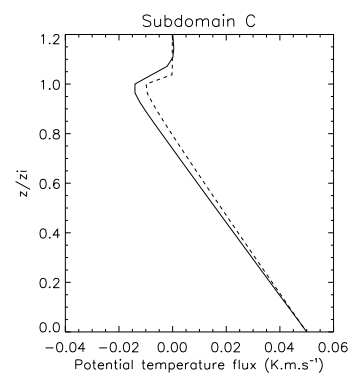

Fig. 2. Comparison of RANS (dashed line) and LES (continuous line) dynamical variables. Vetical profiles of (a) total wind speed, (b) potential temperature, (c) Turbulent kinetic energy, (c) turbulent heat flux. The vertical coordinate is normalised by the boundary layer height (zi) 


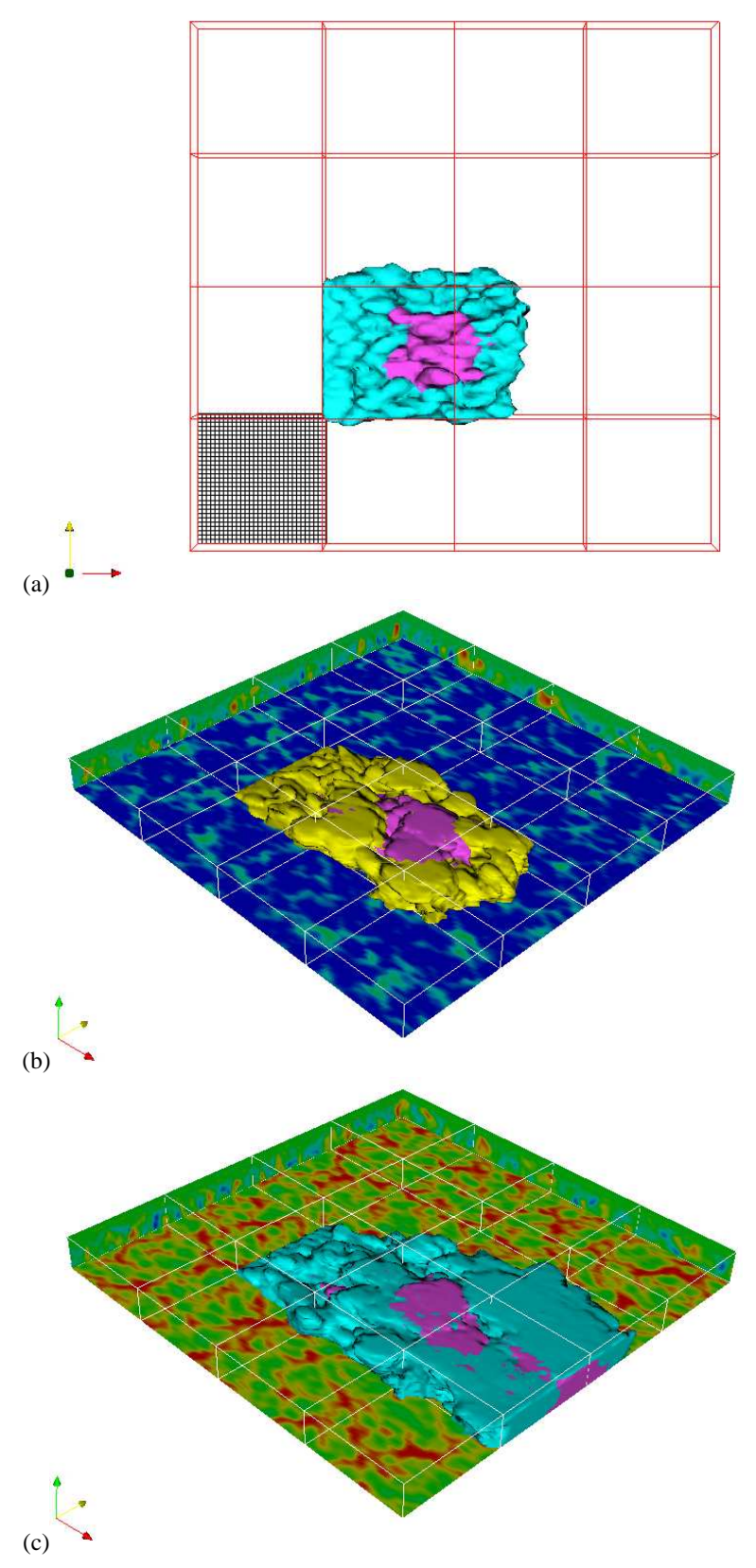

Fig. 3. Snapshots of the tracer dispersion ( $5 \mathrm{~min}$ after the release start) as from the Large-Eddy simulation. (a) top view of the releases from the two surfaces namely $100 \%$ of the RANS grid cell (blue), $28 \%$ of the RANS grid cell (purple). In (a) the grid corresponds to the RANS grid and the LES sub-domains over which LES results have been averaged. In the lowest left corner a sample of the actual LES grid. (b) and (c) two different stages of the tracers dispersion at $10 \mathrm{~min}$ and $25 \mathrm{~min}$ respectively after the release start. Colors on the bottom layers correspond to turbulent heat flux and on vertical wall to vertical velocity.

\section{Sub-grid scale emission: evaluation of the parametrization}

The simplest way to create a sub-grid scale emission heterogeneity is to consider a single area source emitting within one of the RANS cells that varies in size from total coverage of the cell to the minimum resolvable size for the LES. Three different emission scenarios were simulated. For the first the passive tracer is released over the entire RANS grid element (see Fig. 1). The remaining three cover the grid elements corresponding to 64,44 and $28 \%$ of the grid surface thus leading to an increasing sub-grid scale variability of the emission and corresponding emission variance. The tracer released has initially zero concentration. Each emitting surface releases continuously with an emission rate of $0.1 \mathrm{ppbs}^{-1}$. The flux is maintained constant in spite of the change in the size of the emitting surface. Non-periodic boundary conditions are used for the tracer. The simulations run for $3 \mathrm{~h}$ for the dynamic and $2 \mathrm{~h}$ for the tracer dispersion. In the LES non-periodic boundary conditions are set up for the scalars. All the results relate to the last hour of the simulation. Figure 3 a through $\mathrm{c}$ represents snap shots of the evolution of the dispersion process as simulated by the LES. Figure 3a shows a top view of the largest emission pattern (blue contour) and the smallest (magenta contour) after $5 \mathrm{~min}$ from the release start. The figure shows also the RANS grid (in red) and for one RANS grid cell the LES grid (in black). From the figure one can appreciate the difference in size between the two scenarios, the scales involved in the dispersion process, and the scales falling into a RANS grid that would not be resolved by the RANS model. Figure $3 \mathrm{~b}$ and $\mathrm{c}$ shows the subsequent stages of evolution of the emissions at 10 and $25 \mathrm{~min}$ from release start. The contours on the bottom of the simulation domain relate to surface temperature while on the domain walls they relate to vertical velocity.

Figure 4a shows the mean concentration profiles calculated in the six RANS cells (6 LES sub domains). The results of RANS (dashed line) remain the same in spite of the variability of the emitting scenario since the average emission rate remains unchanged. The LES results (continuous line) vary from one case to the other. Most of the mass is concentrated in the emitting cell (cell C) and is advected eastward. A small amount of mass is predicted in cells A and B by the RANS model due to the small discrepancies in the wind field prediction. The average concentration behavior can also be considered comparable among the two models having considered the fact the RANS model at this level does not take into account the sub-grid scale variability of the emission intensity. This is as far as any mesoscale transport model can get: modeling the mean concentration evolution from the mean emission rate. The discrepancies in predicting the mean concentration are largely due to the coarse resolution of the RANS model. Figure $4 \mathrm{~b}$ shows the same calculation performed for Fig. 4a but with a $1 \mathrm{~km} \times 1 \mathrm{~km}$ grid resolution. The results improve systematically. The reason for the selection 

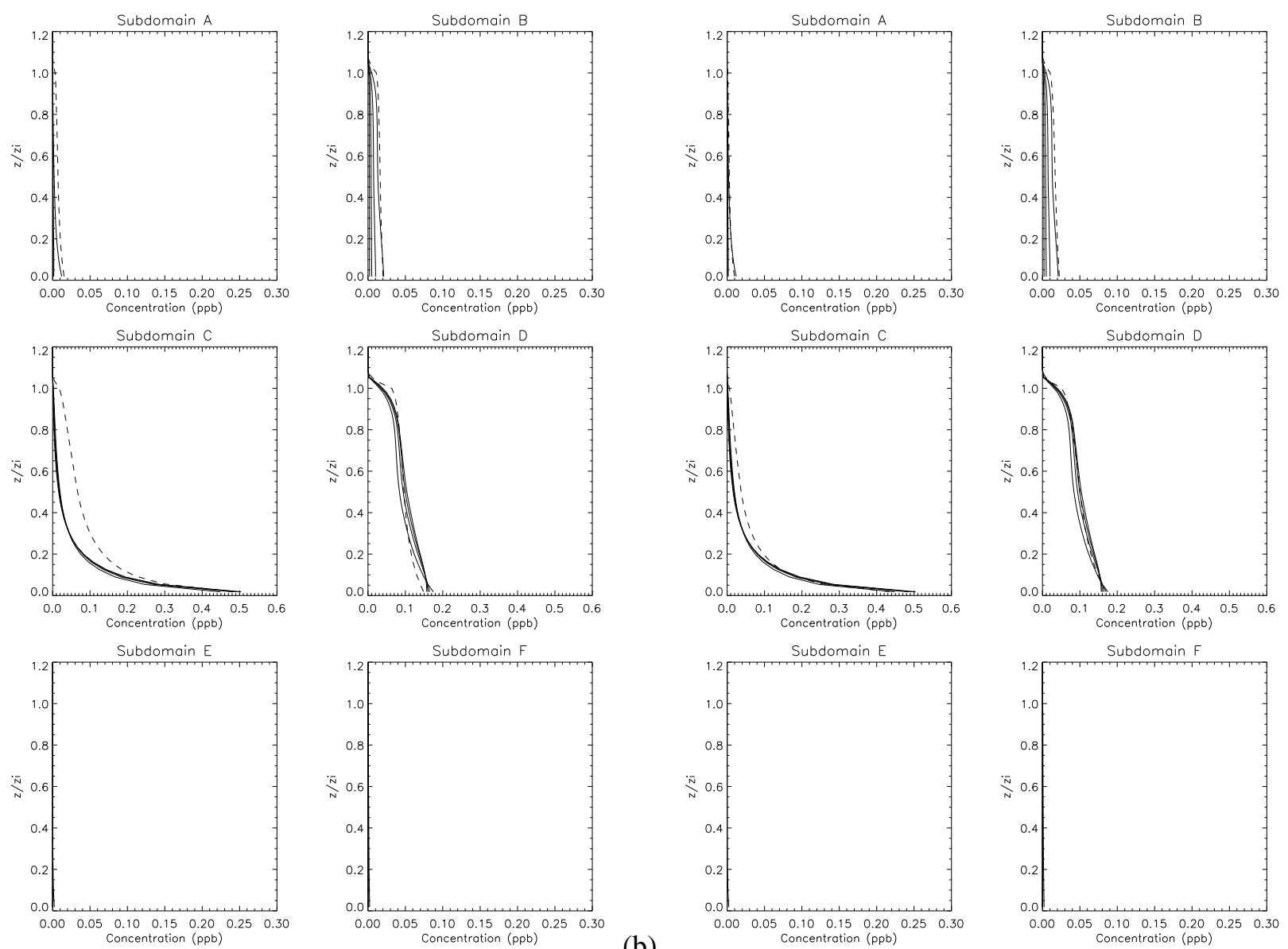

(a)

(b)

Fig. 4. (a) Vertical profiles of the tracers mean concentration in the 6 grid cells labeled a through $\mathrm{f}$ and depicted in Fig. 1. The vertical coordinate is normalized by the boundary layer height. Continuous line LES results, dashed line RANS model. The results relate to the $3 \times 3 \mathrm{Km}$ RANS model resolution. (b) same as (a) but with RANS model running at $1 \times 1 \mathrm{Km}$ resolution.

of the coarse resolution of $3 \times 3 \mathrm{~km}^{2}$ is twofold. As anticipated earlier, first we wanted to test the parametrization on a mesoscale average grid size; secondly $1 \times 1 \mathrm{~km}^{2}$ would have reduced the surface emission size to the extent that also in the LES only few cells would have been available to simulate the release thus reducing the accuracy of the calculation.

Let us now consider the new parametrization to account for the emission heterogeneity within the RANS model. In order to implement our parametrization in the RANS model we still have the unsolved problem of the closure of the emission-concentration correlation coefficient introduced in Sect. 2. We will make use here of the high resolution simulations to study the correlation coefficient behavior and try to find a simple functional relationship to assign it a value. Figure 5 shows the variation of the correlation coefficient between the emission and the concentration calculated explicitly from the LES model as a function of the horizontal dimension of the emitting surface. Not unexpectedly the coefficient tends to zero as the surface shrinks and to one as it widens. The correlation coefficient has been calculated also for other surface sizes than the four analysed here. Although the trend appears to be linear it is expected to flatten toward 1 in a sort of square-root trend. Figure 6 shows the variability of the correlation coefficient as a function of height assuming therefore that the first computational grid point sits at $12.5 \mathrm{~m}$ (squares), $37.5 \mathrm{~m}$ (triangle), $62.5 \mathrm{~m}$ (diamond), $87.5 \mathrm{~m}$ (star) and $112.5 \mathrm{~m}$ (cross). As it can be seen in all cases the correlation coefficient shows a well behaved trend that can easily be framed in a functional relationship. However we will not do this in this study as we realise that a dedicated research will need to be performed on the subject toward the definition of a general formulation. For the sake of this study we will use the coefficient values extracted from the plot of Fig. 5 as we still want to prove that the parameterization is valid. A 


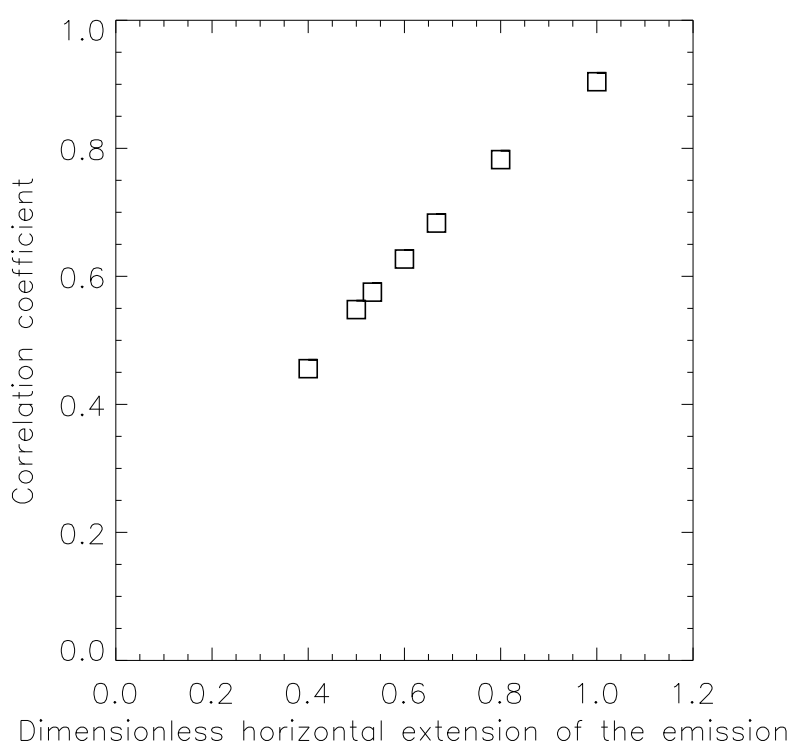

Fig. 5. Correlation coefficient of concentration and emission as a function of the horizontal extension of the emitting surface.

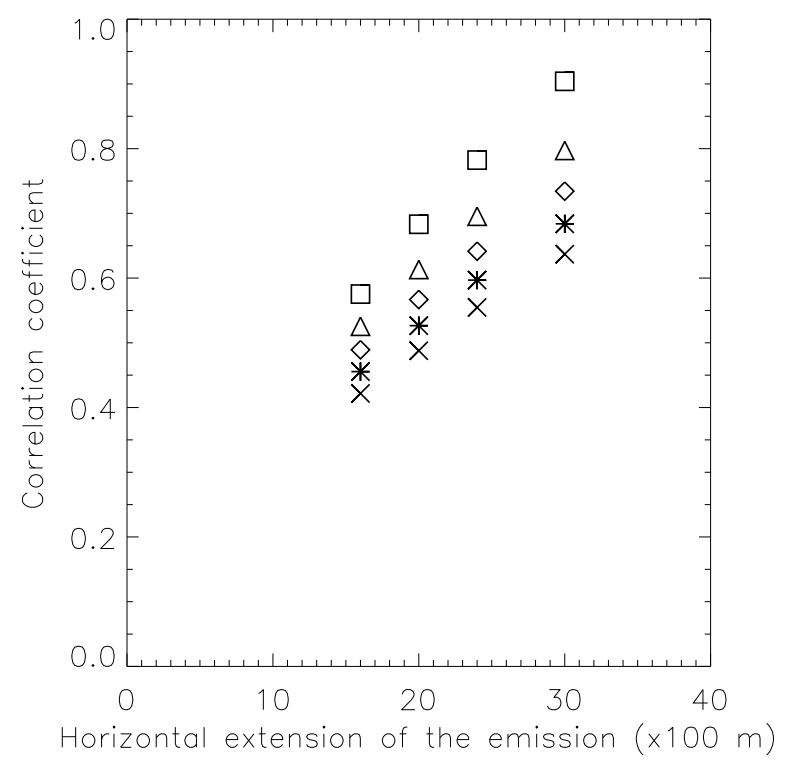

Fig. 6. Same as Fig. 5. The different samples give the dependence of the correlation coefficient on height. Squares $12.5 \mathrm{~m}$ from the surface, triangles $37.5 \mathrm{~m}$, diamond $62.5 \mathrm{~m}$, star $87.5 \mathrm{~m}$, cross $112.5 \mathrm{~m}$.

sensitivity analysis on the impact of an approximated value on the performance of the parametrization will need to be performed as well as its dependence on wind speed, heat flux and height.
Having identified a correlation coefficient we can close our parametrization and calculate the concentration variance produced by the emission variability. Figures 7,8 and 9 show the vertical profile of concentration variance calculated by the RANS model and the LES (continuous line) for the 64, $44,28 \%$ values of emission surface to grid cell surface ratio respectively. The parametrization seems to work extremely well in the emission cell where most of the variance is produced in correspondence with the largest concentration gradients. This is valid also for the other two cases with a slight overestimation of the values through out the profile. In all three cases in cell D the values in the bulk of the boundary layer are very well reproduced, while a large discrepancy is present close to the surface that can be attributed to an excess in dissipation of the RANS formulation. The differences are more marked as the emitting surface shrinks. In cells A and $\mathrm{B}$ for all the three cases we notice an over prediction by the RANS model that can be connected to the fact that the latter predicts a non zero concentration in those two cells as described earlier. In all the cases we notice that the discrepancies are there but pertain to values of concentration variance that is one order of magnitude smaller than in the emitting cell. It is in fact interesting to notice that the dissipation of concentration variance takes place over a relatively short time scale thus not permitting its advection to the neighboring cells as also confirmed by the LES results. In the vertical the values are relevant in the first half of the boundary layer and decrease rapidly from there to the its top. All the results described above are corroborated and emphasised by the analysis of the variance calculated without taking into account the new production term (10) and presented in Figs. 7, 8 and 9 by the thin dashed line. This is particularly evident for the small surface (Fig. 9). The figure clealy shows the relevance of the variance in the vertical in the emission cell and its advection that in subdomains A, B and D accounts for almost double of the variance without emission contribution.

A direct evidence of the impact of emission heterogeneity to boundary layer concentration can be obtained by sampling the LES domain at specific points in time. We will call these point virtual monitoring stations since they will behave like sampling stations in the real atmosphere which can have only a partial view of the process but can describe it in great detail in time. Figure 10 shows the location of the station (a) and two time series (b and c) for the $64 \%$ percent emission surface (depicted in (a) as instantaneous concentration contour) and station (d) and the corresponding time series for the $24 \%$ emission (e and f). Figure $10 \mathrm{~b}$ and e refers to the comparison of the one minute averaged concentration time series from the LES (red curve) and RANS (black curve). Figure 10c and $f$ shows the comparison of the 5 min average concentration time series from the two models. In both figures the shaded area covers the $\bar{c} \pm \sqrt{\overline{c^{\prime 2}}}$ where the standard deviation is the result of the parametrization within the RANS model. The results show a remarkable correspondence between the 


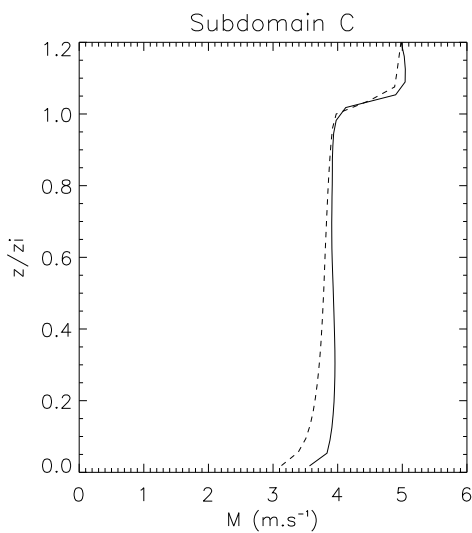

(a)

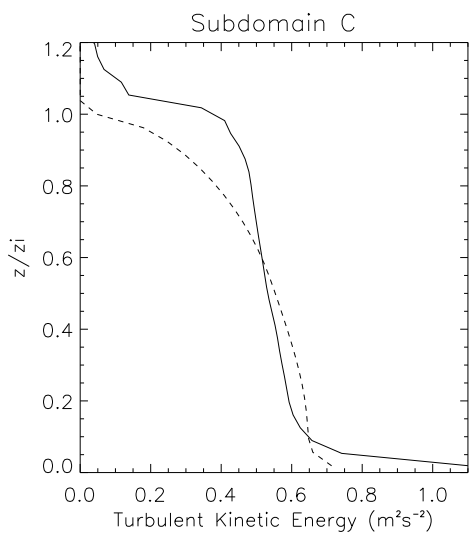

(b)
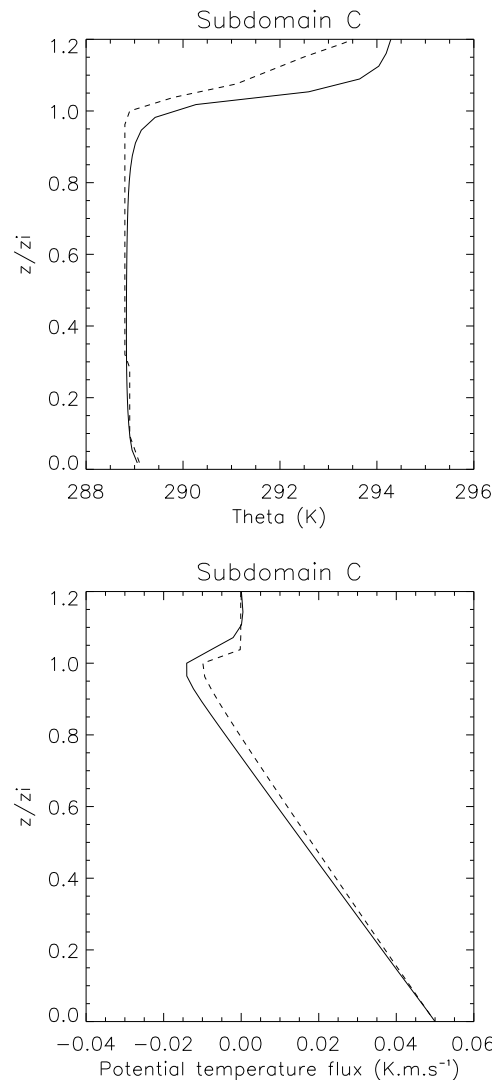

(c)

(d)

Fig. 7. Comparison of the vertical profile of concentration variance calculated by the RANS (thick dashed line) and LES (thick continuous line) models. The vertical coordinate is normalized by the boundary layer height. The results relate to the RANS model grid cells and corresponding LES sub-domains depicted in Fig. 1. The emission surface is chosen to be $64 \%$ of the RANS grid size. In all subdomains, the thin dashed line represents the concentration variance calculated without the contribution of the new emission production term in the variance equation

RANS average concentration plus the standard deviation and the LES time series for both cases. A slight underestimation of the LES results is shown by the RANS mean concentration but all fluctuations fall well within the standard deviation. The same is true for the other station selected for the emission domain and shown in Fig. 11a-f. In particular in the latter we see that most of the deviation is due to the lack of agreement in the average concentration but that the bandwidth of the standard deviation covers all the RANSsub-grid fluctuations explicitly calculated by the LES. Moving to the grid-cells downwind (Figs. 12-14a-f) of the emission we notice, that still the variance has a relevant role in catching the sub-grid fluctuation. One can also notice the variability of the variance by comparing the large surface emission with the smallest. In all three virtual monitoring stations the mesoscale model is not able to catch the quasiinstantaneous results of the LES which is very dependent on where the cloud shows up with respect to the station (see
Fig. 3 for example) but when the data are time averaged to longer time scales, the RANS variance and its use is making the difference in for the interpretation of the results. It should be underlined that the case simulated is extremely complicated for any RANS model especially at these resolutions. One should not forget that the ratio of resolution of the two models is 960/864000 and disregarding the fact that the two models have the same vertical resolution the RANS with the new parametrization models with 16 grid cells what the LES models with 14400 .

\section{Conclusions}

A method to account for the sub-grid emission heterogeneity has been proposed. The method is based on the modification of the concentration variance equation and the assumption that emission can be linearized in an average and a fluctuating part and a correlation between emission and air 

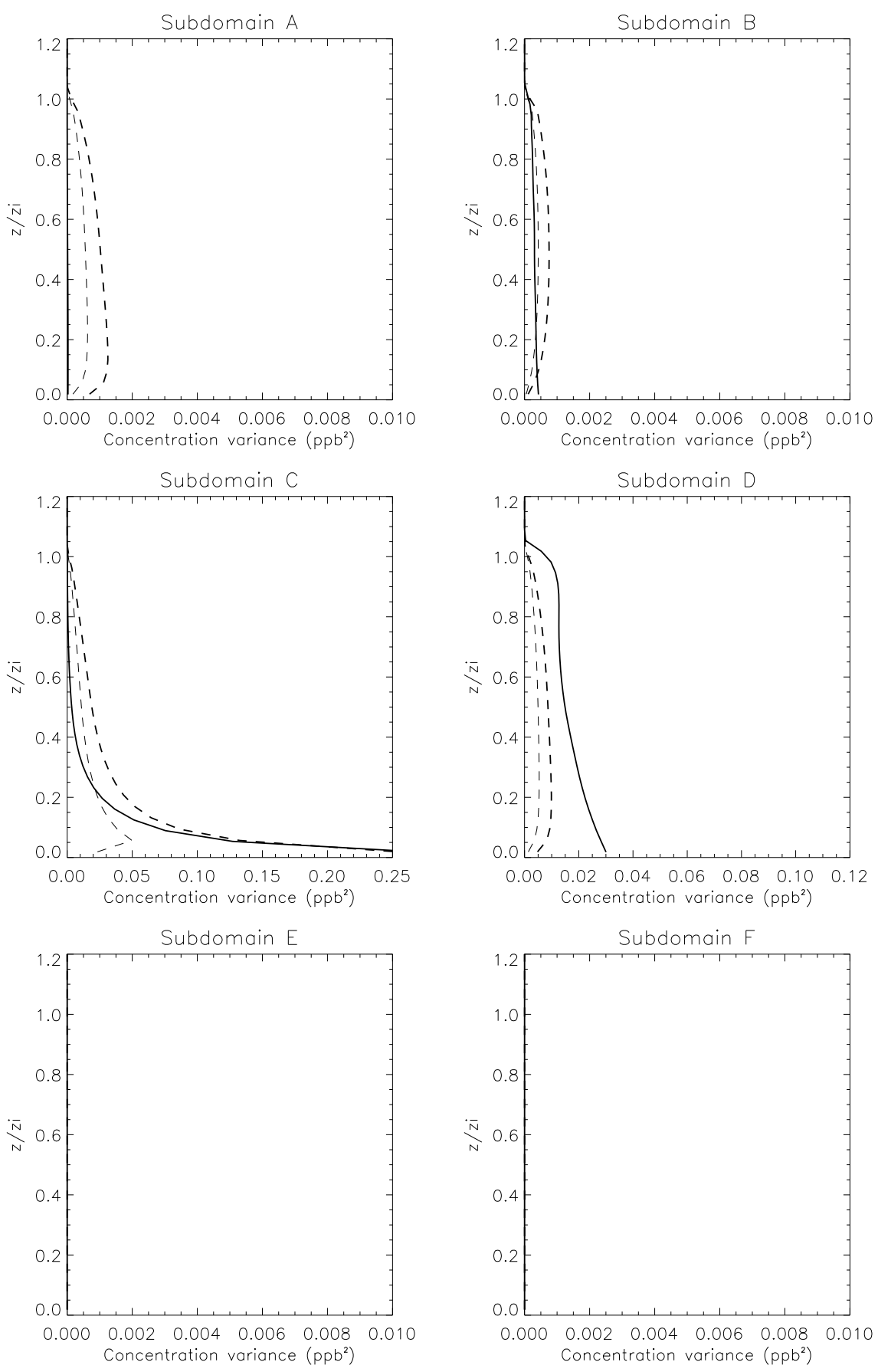

Fig. 8. Same as Fig. 7. The results relate to the emission surface of $44 \%$ of the RANS grid size.

concentration within the model grid cell. The ingredients for the application of the parametrization is the availability of an emission inventory that can provide disaggregated values at a scale smaller that the grid used in the transport model. The method allows one to explicitly calculate the time evolution of the concentration variance in every grid cell, its transport and dissipation as well as the contribution of the emission variability at the surface to its creation. The parametrization presented in this paper can be applied to any model from meso- to global scale. In fact the assumption normally made in these two kind of models with respect to emission treatment are the same. For the first time, the method proposed 

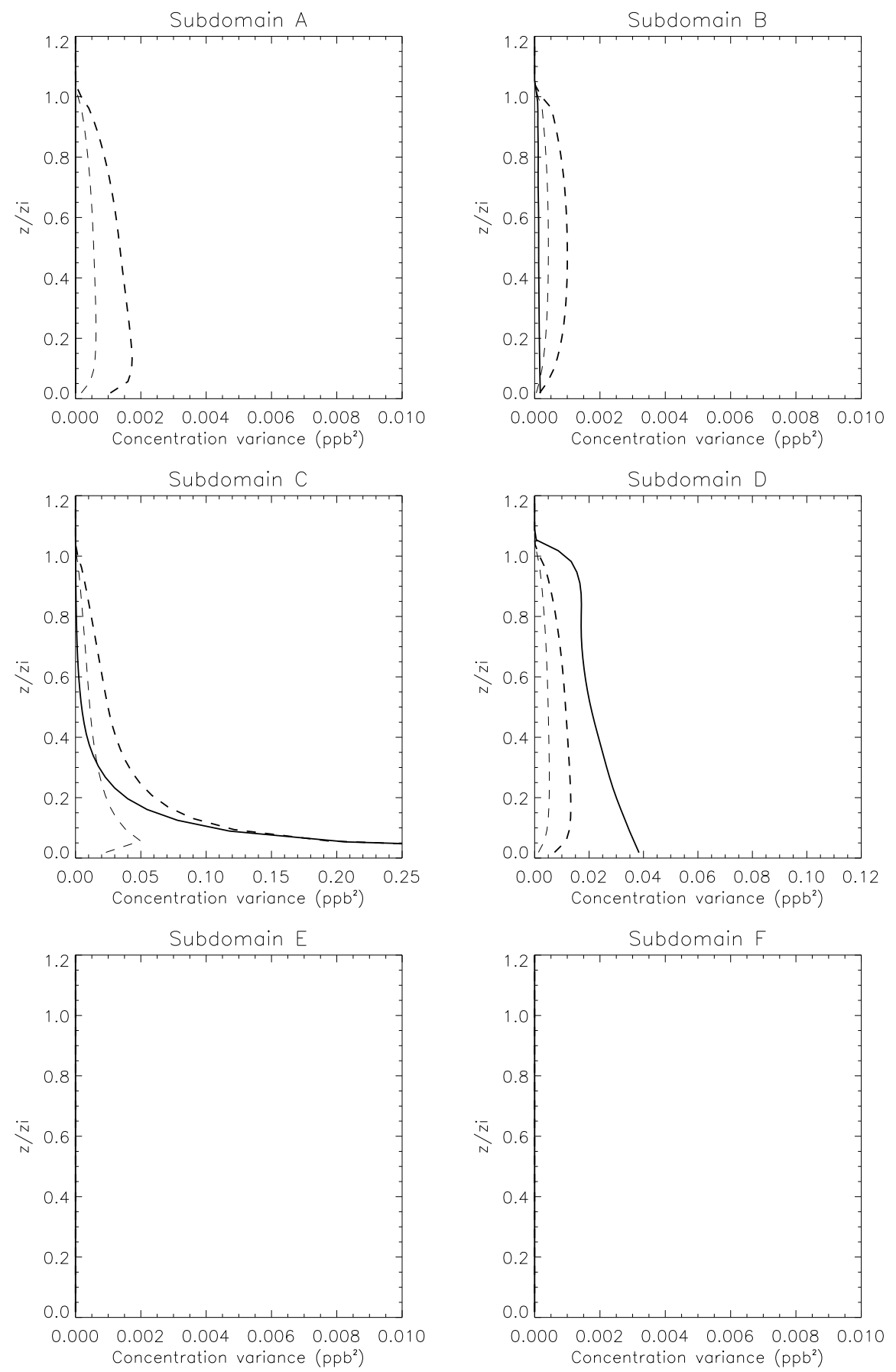

Fig. 9. Same as Fig. 7. The results relate to the emission surface of $28 \%$ of the RANS grid size.

allows to calculate explicitly the time evolution of the subgrid variability of a variable and to attach it to the mean concentration value. The parametrization has been made part of a mesoscale model and the results on a variety of cases compared with high resolution simulation performed with a Large Eddy Simulation model. The results show a very satisfactory agreement.
More generically the concept developed here could be applied in several other contexts like:

1. Homogeneous emission of inert or reacting scalars. In this case the new term of the concentration variance equation would vanish but still there would be an impact of the concentration variance equation which has never 


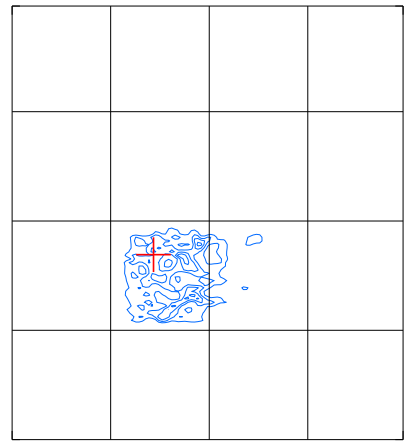

(a)

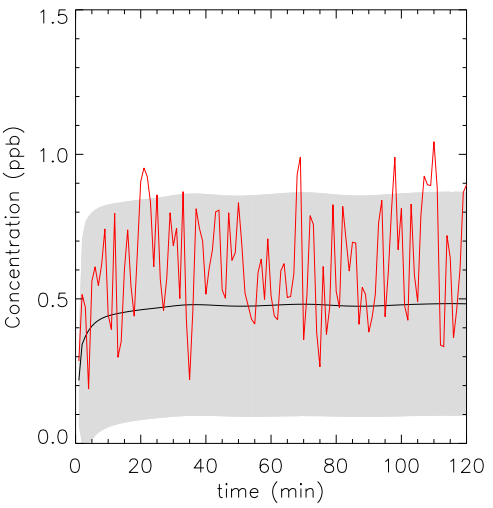

(b)

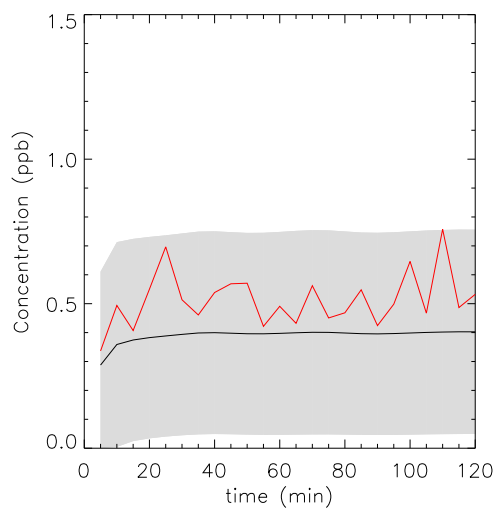

(c)

(f)

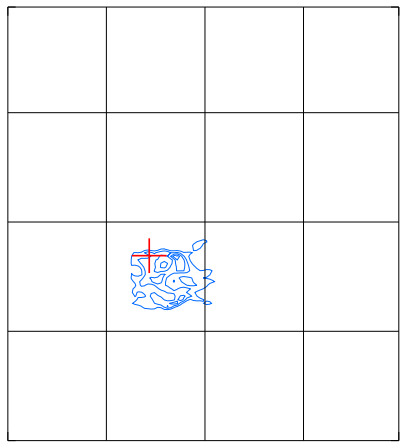

(d)

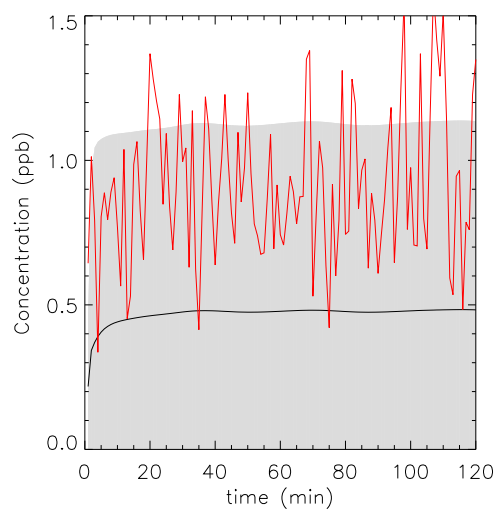

(e)

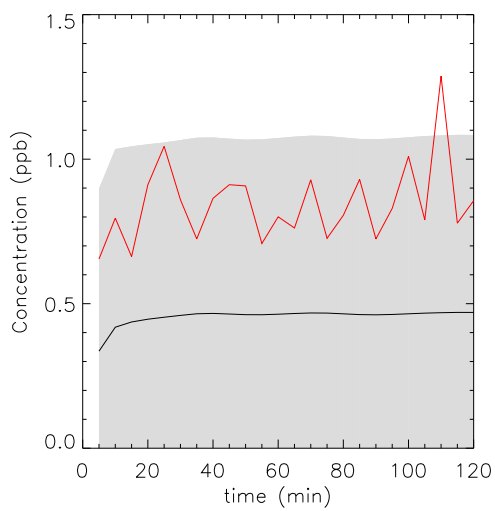

Fig. 10. (a and d) Emission scenarios and position of the virtual station with respect to the RANS grid. (a) 64\% surface emission, (d) $28 \%$ surface emission. (b and e) comparison of the time evolution of the RANS concentration (continuous line) plus and minus standard deviation (hatched surface) with the concentration from the LES (red line) both model results are averaged in time over 1 min. (c and $\mathbf{f}$ ) same as (b and e) but with model results averaged over $5 \mathrm{~min}$.

been accounted for. The concentration variance is a statistical representation of the effect of inhomogeneous turbulent mixing. Therefore regardless of the modification introduced in this paper to the variance equation, it could be used to account for the sub-grid mixing. To date, no mesoscale model has taken explicitly into ac- count this element. Indeed the effect is relevant within the boundary layer, which is a small portion of the atmosphere represented by a global model for example, but yet there is where the comparison with surface measurements takes place. 


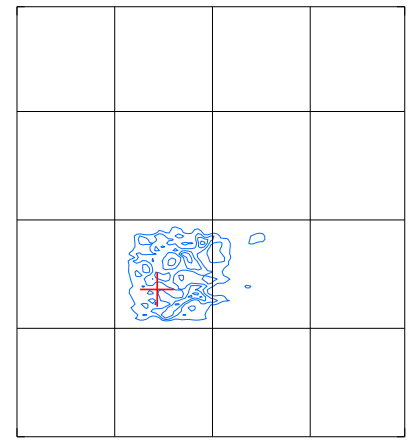

(a)

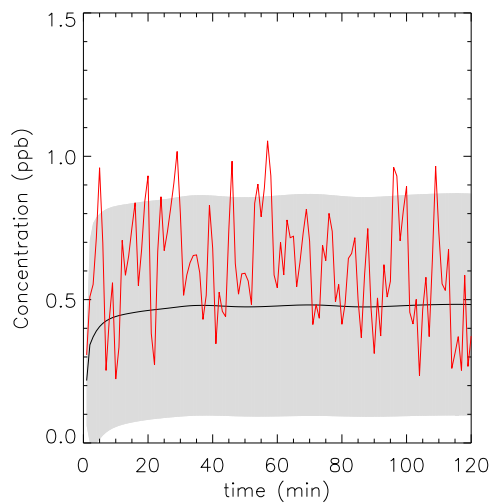

(b)

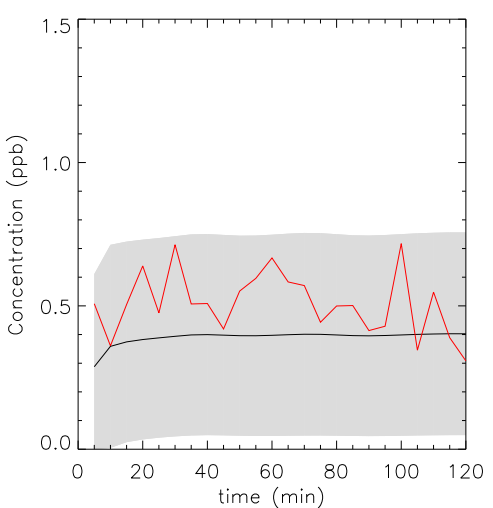

(c)

Fig. 11. Same as Fig. 10 but different sampling virtual station.

2. The method proposed here could be applied to inert scalars in-homogeneously emitted at the surface like for example heat or moisture emitted from the surface.

An element of concern could be the fact that every species will require an additional equation to be solved and that it may constitute a burden for large chemical scheme, however we could consider to use it only to primary pollutants. Furthermore the solution of the highly parameterized variance equation is straight forward and inexpensive. Another con-

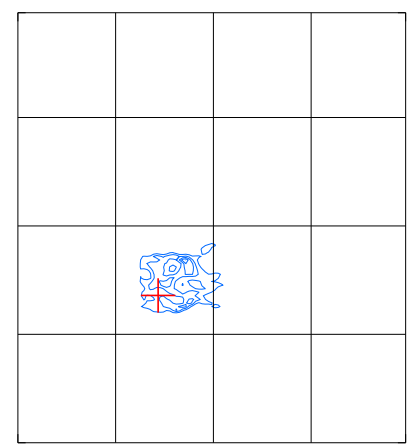

(d)

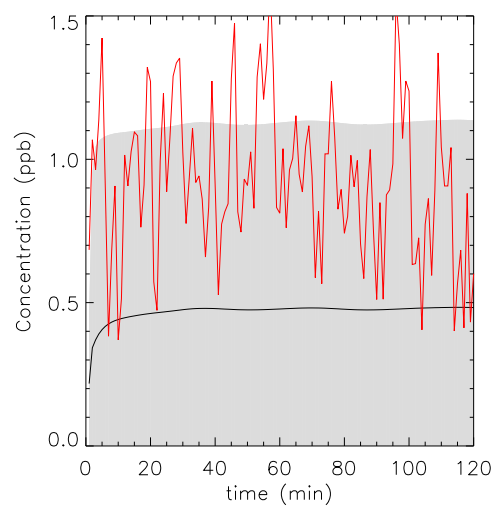

(e)

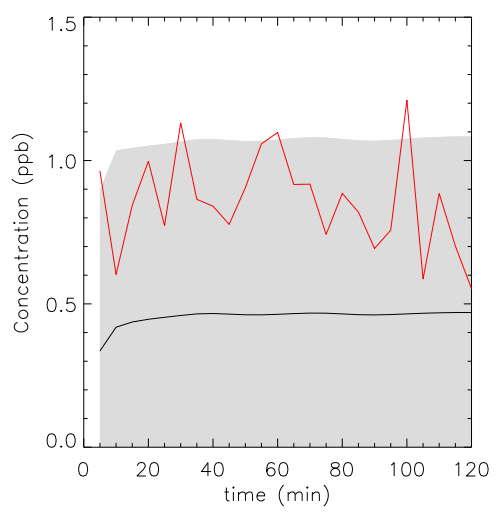

cern may relate to the effect of chemical reaction on the new variable. In other words would the scalar react should we take into account the chemical reaction also at the level of the concentration variance? An answer to this problem has already been given by the large number of studies performed since the 1990ies on the influence of turbulent mixing on chemical reaction in the atmosphere (e.g., Sykes et al., 1994; Galmarini et al., 1997; Verver et al., 1997; Vinuesa and VilàGuerau de Arellano, 2003, 2005). These works have shown that the discrimination in the application of chemical reaction 


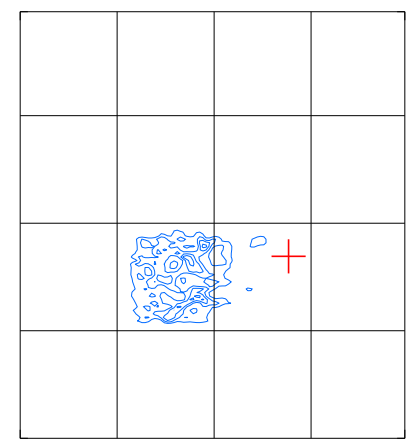

(a)

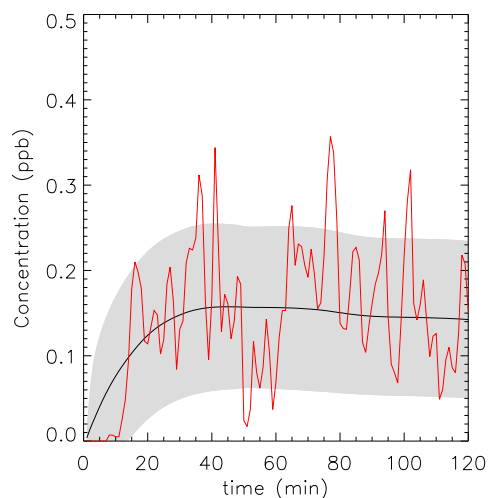

(b)

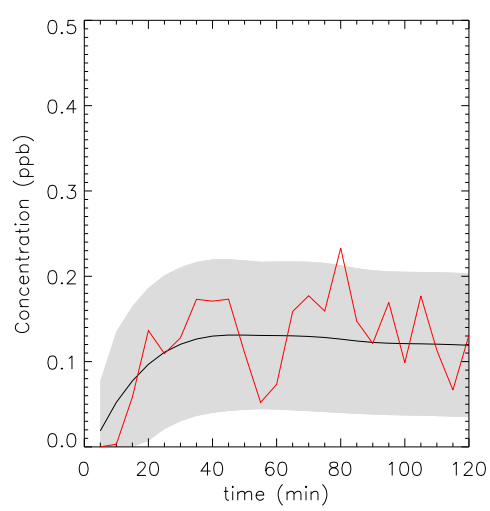

(c)

Fig. 12. Same as Fig. 10 but different sampling virtual station.

to the second order model equation is the ratio of the time scale of turbulence and that of chemistry. A finite number of species in the atmosphere fulfill this requirement and only for those chemistry should be taken into account.

The next steps of our research will concentrate on the following aspects:

1. frame in the most general way the correlation coefficient between emission and concentration in air,

2. verify the sensitivity of the parametrization on the vertical resolution of the mesoscale model,

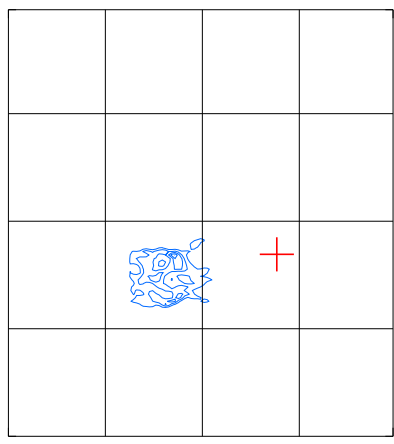

(d)

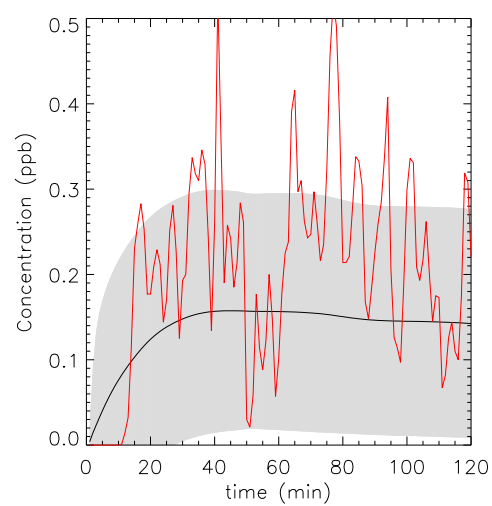

(e)

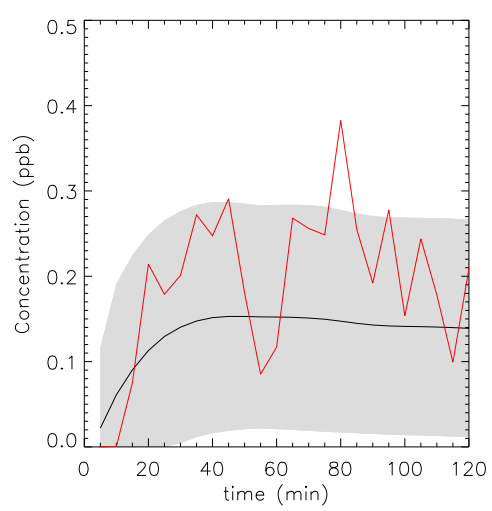

(f)

3. apply the mesoscale model to a real case study,

4. develop a parametrization to account for the sub-grid topological orientation of the emission heterogeneity.

In this last case the only possibility to test the parametrization will be by comparison of the predicted concentration plus and minus the variance and the measured ones, so only indirectly. Therefore the case will be selected to as dominated by large emission variability. 


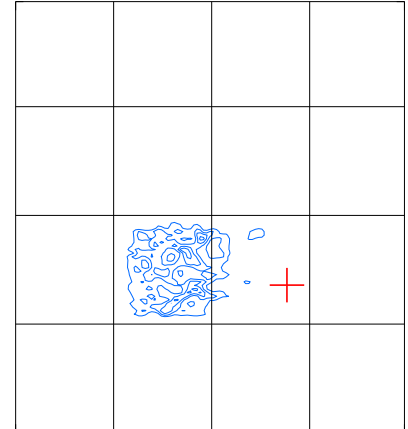

(a)

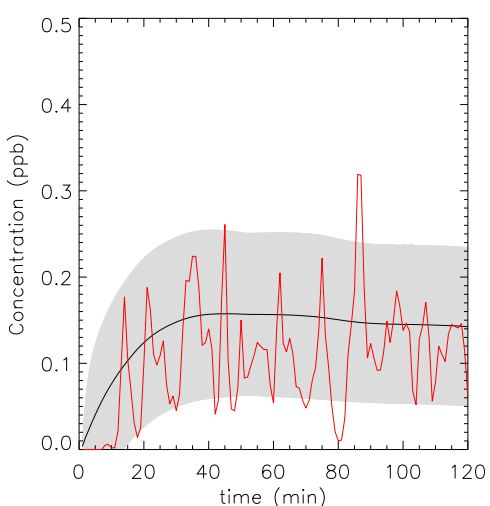

(b)

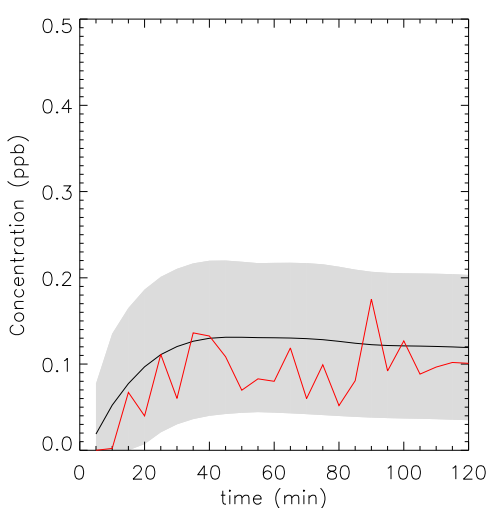

(c)

Fig. 13. Same as Fig. 10 but different sampling virtual station.

\section{Appendix A}

\section{Closures used for prognostic equation of the} variance of pollutant concentration

A description of the other closures used to solve the prognostic equation for the variance (8) is given.

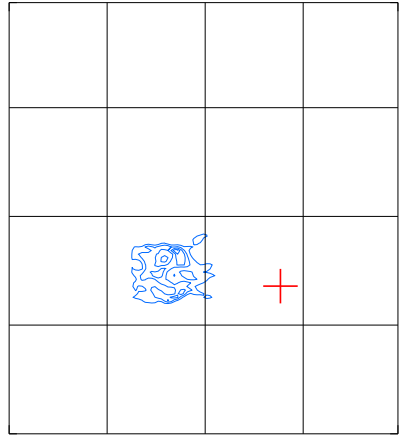

(d)

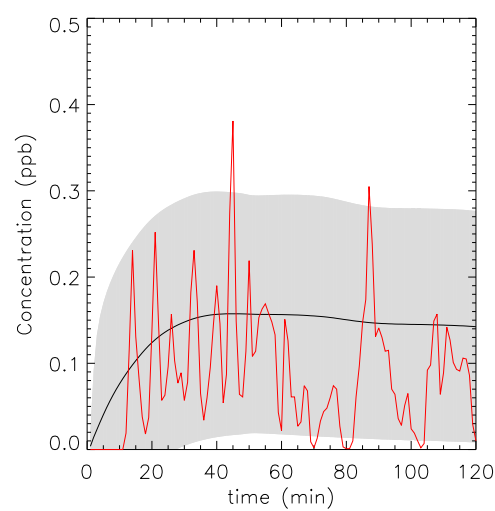

(e)

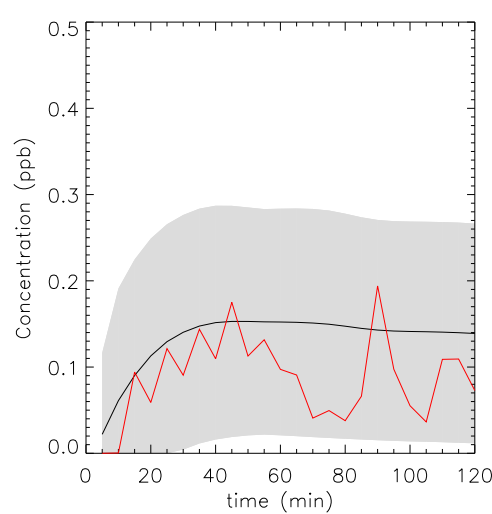

(f)

The turbulent fluxes of (1) are calculated as

$\overline{w^{\prime} c^{\prime}}=-\frac{K_{z}}{\operatorname{Pr}} \frac{\partial \bar{c}}{\partial z}$

where the turbulent coefficient $K_{z}$ is estimated using a K1 closure (Bougeault and Lacarrére, 1989). In this closure a prognostic equation for the turbulent kinetic energy $e$ is solved, and turbulent coefficients and TKE dissipation are 


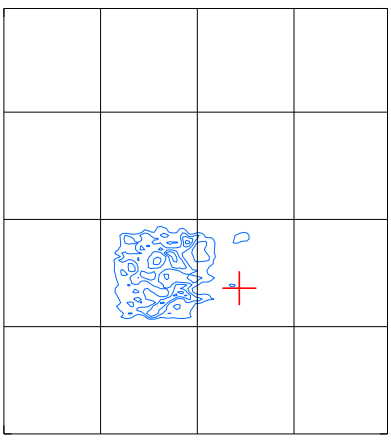

(a)

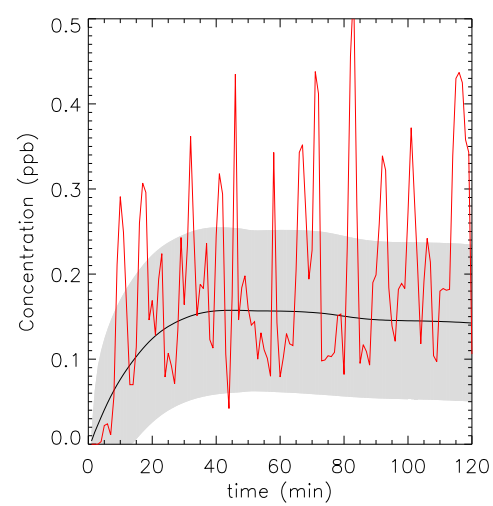

(b)

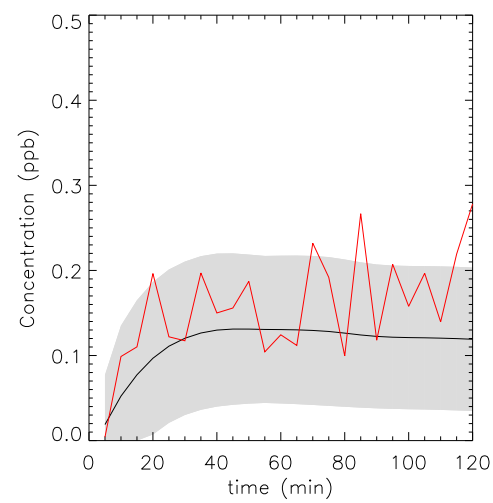

(c)

Fig. 14. Same as Fig. 10 but different sampling virtual station.

derived using length scales as follows:

$K_{z}=C_{k} l_{k} e^{1 / 2}$

$\epsilon_{e}=C_{\epsilon} \frac{e^{3 / 2}}{l_{\epsilon}}$

The lengths $l_{k}$ and $l_{\epsilon}$ are calculated at a particular level from the possible upward and downward displacements ( $l_{\text {up }}$ and $l_{\text {down }}$ ) that air parcels with kinetic energy $e$ originating from

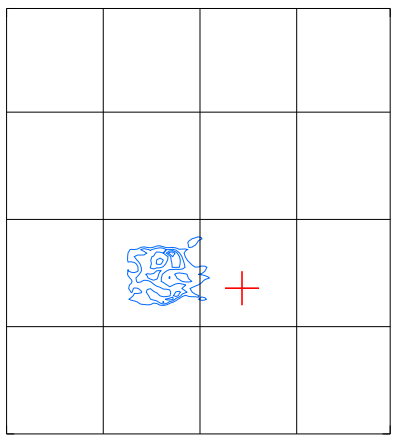

(d)

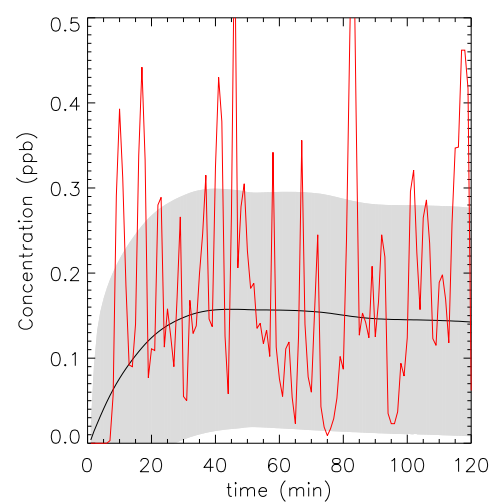

(e)

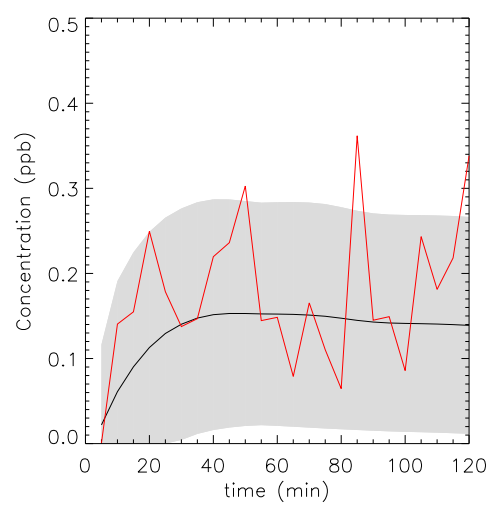

(f)

the level $z$ could accomplish before being stopped by buoyancy.

$$
\begin{aligned}
& \int_{z}^{z+l_{\mathrm{up}}} \beta\left(\theta(z)-\theta\left(z^{\prime}\right)\right) d z^{\prime}=e(z), \\
& \int_{z-l_{\mathrm{down}}}^{z} \beta\left(\theta\left(z^{\prime}\right)-\theta(z)\right) d z^{\prime}=e(z),
\end{aligned}
$$

Therry and Lacarrére (1983) proposed a relationship between 
$l_{\epsilon}$ and $l_{k}:$

$l_{k}=\left(1+\frac{g}{\theta} \frac{\overline{w \theta} l_{\epsilon}}{C_{\epsilon} e^{3 / 2}} l_{\epsilon}\right) l_{\epsilon}$

(Bélair et al., 1999) used the budget equation for the TKE to derive the relationship neglecting the turbulent transport contribution and assuming steady-state. This leads to

$l_{k}=\left(1+\frac{B_{e}}{D_{e}}\right) l_{\epsilon}$

or

$l_{k}=\left(\frac{2 B_{e}+G_{e}}{B_{e}+G_{e}}\right) l_{\epsilon}$

where $B_{e}, D_{e}$ and $G_{e}$ are the buoyancy, the dissipation and the gradient terms of the TKE budget equation. $l_{k}$ is determined as the minimum between $l_{\text {up }}$ and $l_{\text {down }}$ (Bougeault and Lacarrére, 1989).

The turbulent transport of variance can be written as

$\frac{\partial \overline{u_{j} c^{\prime 2}}}{\partial x_{j}}=-\frac{\partial}{\partial z}\left(\frac{K_{z}}{\operatorname{Pr}} \frac{\partial \overline{c^{\prime 2}}}{\partial z}\right)$

The dissipation can be written as

$2 \epsilon \overline{c^{\prime 2}}=\frac{\overline{c^{\prime 2}}}{\tau \overline{c^{\prime 2}}}$

(Verver et al., 1997) used the TKE dissipation timescale divided by 2.5 as variance dissipation timescale to be inserted in the expression of the scalar variance dissipation. Using this expression together with Eq. (A3) leads to

$\epsilon \overline{c^{\prime 2}}=2.5 C_{\epsilon} \frac{e^{1 / 2}}{l \epsilon} \overline{c^{2}}$

$C_{\epsilon}$ and $C_{k}$ are set to 0.125 and 0.7 and the Prandtl $P r$ number is $1 / 1.3$. Boundary conditions for the TKE and the variances are calculated assuming no gradients across the surface.

Acknowledgements. All computations were performed on the Linux cluster of the PROCAS action of the Institute for Environment and Sustainability. The authors wish to thank A. Stips and P. Simons who kindly provided the access to this facility.

Edited by: J. Quaas

\section{References}

Bélair, S., Mailhot, J., Strapp, J. W., and MacPherson, J. I.: An examination of local versus nonlocal aspects of a TKE-based boundary-layer scheme in clear convective conditions, J. Appl. Meteorol., 38, 1499-1518, 1999.
Beare, R. J., Macvean, M. K., Holtslag, A. A. M., Cuxart, J., Esau, I., Golaz, J.-C., Jimenez, M. A., Khairoutdinov, M., Kosovic, B, Lewellen, D., Lund, T. S., Lundquist, J. K., Mccabe, A., Moene, A. F., Noh, Y., Raasch, S., and Sullivan, P.: An intercomparison of large-eddy simulations of the stable boundary layer, Bound.Lay. Meteorol., 118, 247-272, 2006.

Bougeault, P. and Lacarrére, P.: Parameterization of orographyinduced turbulence in a mesobeta-scale model, Mon. Weather Rev., 117, 1872-1890, 1989.

Cuijpers, J. W. M. and Duynkerke, P. G.: Large eddy simulations of trade wind with cumulus clouds, J. Atmos. Sci., 50, 3894-3908, 1993.

Cuijpers, J. W. M. and Holtslag, A. A. M.: Impact of skewness and nonlocal effects on scalar and buoyancy fluxes in convective boundary layers, J. Atmos. Sci., 55, 151-162, 1998.

Dosio, A., Vilà-Guerau de Arellano, J., Holtslag, A. A. M., and Builtjes, P. J. H.: Dispersion of a passive tracer in buoyancyand shear-driven boundary layers, J. Appl. Meteorol., 42, 11161130, 2003.

Dosio, A., Vilà-Guerau de Arellano, J., Holtslag, A. A. M., and Builtjes, P. J. H.: Relating eulerian and lagrangian statistics for the turbulent dispersion in the atmospheric convective boundary layer, J. Atmos. Sci., 62, 1175-1191, 2005.

Dosio, A. and Vilà-Guerau de Arellano, J.: Statistics of absolute and relative dispersion in the atmospheric convective boundary layer: a large-eddy simulation study, J. Atmos. Sci., 63, 12531272, 2006.

Galmarini, S., Vilà-Guerau de Arellano, J., and Duynkerke P. G.: Scaling the turbulent transport of chemical compounds in the surface layer under neutral and stratified conditions, Q. J. R. Meteorol. Soc., 123, 223-242, 1997.

Galmarini, S., Beets, K., and Duynkerke, P. G.: Stable nocturnal boundary layers: a comparison of one-dimensional and largeeddy simulation models, Bound.-Lay. Meteorol., 88, 181-210, 1998.

Garratt, J. R.: The atmospheric boundary layer, Cambridge University Press, 316 pp., 1994.

Jonker, H. J. J., Vilà-Guerau de Arellano, J., and Duynkerke, P. G.: Characteristic length scales of reactive species in a convective boundary layer, J. Atmos. Sci., 61, 41-56, 2004.

Martilli, A.: Numerical study of urban impact on boundary layer structure: sensitivity to wind speed, urban morphology, and rural soil moisture, J. Appl. Meteorol., 41, 1247-1266, 2002.

Meeder, J. P. and Nieuwstadt, F. T. M.: Large-eddy simulation of the turbulent dispersion of a reactive plume from a point source into a neutral atmospheric boundary layer, Atmos. Environ., 34, 3563-3573, 2000.

Molemaker, M. J. and Vilà-Guerau de Arellano, J.: Turbulent control of chemical reactions in the convective boundary layer, J. Atmos. Sci., 55, 568-579, 1998.

Neggers, R. A. J., Jonker, H. J. J., and Siebesma, A. P.: Size statistics of cumulus cloud populations in large-eddy simulations, J. Atmos. Sci., 60, 1060-1074, 2003.

Nieuwstadt, F. T. M.: A large-eddy simulation of a line source in a convective atmospheric boundary layer - I, dispersion characteristics, Atmos. Environ., 26A, 485-495, 1992a.

Nieuwstadt, F. T. M.: A large-eddy simulation of a linesource in a convective atmospheric boundary layer - II, dynamics of a buoyant line source, Atmos. Environ., 26A, 497-503, 1992b. 
Siebesma, A. P. and Cuijpers, J. W. M.: Evaluation of parametric assumptions for shallow cumulus convection, J. Atmos. Sci., 52, 650-666, 1995.

Siebesma, A. P. and Holtslag, A. A. M.: Model impacts of entrainment and detrainment rates in shallow cumulus convection, J. Atmos. Sci., 53 2354-2364, 1996.

Siebesma, A. P., Bretherton, C. S., Brown, A., Chlond, A., Cuxart, J., Duynkerke, P. G., Jiang, H., Khairoutdinov, M., Lewellen, D., Moeng, C. H., Sanchez, E., Stevens, B., and Stevens, D. E.: A large eddy simulation intercomparison study of shallow cumulus convection, J. Atmos. Sci., 60, 1201-1219, 2003.

Stull, R. B.: An introduction to boundary-layer meteorology, Kluwer Academic Publishers, 670 pp., 1988.

Sykes, R. I., Parker, S. F., Henn, D. S., and Lewellen, W. S.: Turbulent mixing with chemical reactions in the planetary boundary layer, J. Appl. Meteorol., 33, 825-834, 1994.

Therry, G., and Lacarrére, P.: Improving the eddy kinetic energy model for planetary boundary layer description, Bound.-Lay. Meteorol., 25, 63-88, 1983.

van Zanten, M., Duynkerke, P. G., and Cuijpers, J. W. M.: Entrainment parameterizations in convective boundary layers, J. Atmos. Sci., 56, 813-828, 1999.

Verver, G. H. L., Van Dop, H., and Holtslag, A. A. M.: Turbulent mixing of reactive gases in the convective boundary layer, Bound.-Lay. Meteorol., 85, 197-222, 1997.
Vilà-Guerau de Arellano, J. and Cuijpers, J. W. M.: The chemistry of a dry cloud: the effects of radiation and turbulence, J. Atmos. Sci., 57, 1573-1584, 2000.

Vilà-Guerau de Arellano, J., Dosio, A., Vinuesa, J.-F., Holtslag, A. A. M, and Galmarini, S.: The dispersion of chemically reactive species in the convective boundary layer, Meteorol. Atmos. Phys., 87, 23-28, 2004.

Vilà-Guerau de Arellano, J., Kim, S.-W., Barth, M. C., and Patton, E. G.: Transport and chemical transformations influenced by shallow cumulus over land, Atmos. Chem. Phys., 5, 32193231, 2005, http://www.atmos-chem-phys.net/5/3219/2005/.

Vinuesa, J.-F. and Vilà-Guerau de Arellano, J.: Fluxes and (co)variances of reacting scalars in the convective boundary layer, Tellus, 55B, 935-949, 2003.

Vinuesa, J.-F. and Vilà-Guerau de Arellano, J.: Introducing effective reaction rates to account for the inefficient mixing of the convective boundary layer, Atmos. Environ., 39, 445-461, 2005.

Vinuesa, J.-F. and Galmarini, S.: Characterization of the ${ }^{222} \mathrm{Rn}$ family turbulent transport in the convective atmospheric boundary layer, Atmos. Chem. Phys., 7, 697-712, 2007, http://www.atmos-chem-phys.net/7/697/2007/. 